\title{
Bending and Orientational Characteristics of Long Period Gratings Written in D-Shaped Optical Fiber
}

\author{
T. Allsop, A. Gillooly, V. Mezentsev, T. Earthgrowl-Gould, R. Neal, D. J. Webb, and I. Bennion
}

\begin{abstract}
Long period gratings (LPGs) were written into a D-shaped single-mode fiber. These LPGs were subjected to a range of curvatures, and it was found that as curvature increased, there was increasingly strong coupling to certain higher order cladding modes without the usual splitting of the LPGs stopbands. A bend-induced stopband yielded a spectral sensitivity of $12.55 \mathrm{~nm} \cdot \mathrm{m}$ for curvature and $2.2 \times 10^{-2} \mathrm{~nm}{ }^{\circ} \mathrm{C}^{-1}$ for temperature. It was also found that the wavelength separation between adjacent bend-induced stopbands varied linearly as a function of curvature. Blue and red wavelength shifts of the stopbands were observed as the sensor was rotated around a fixed axis for a given curvature; thus, in principle, this sensor could be used to obtain bending and orientational information. The behavior of the stopbands was successfully modeled using a finite element approach.
\end{abstract}

Index Terms-Curvature measurement, D-shaped optical fiber, long-period fiber gratings, optical fiber devices, temperature measurement.

\section{INTRODUCTION}

A FIBER long period grating (LPG) is an axially periodic refractive index variation inscribed in the core of a photosensitive single-mode optical fiber by ultra-violet irradiation, which couples light from the core of the fiber into the fiber cladding modes at discrete wavelengths. The index modulation change within the core of a single mode optical fiber is approximately $10^{-4}$ and has a period typically between $100-600 \mu \mathrm{m}$. The index modulation produces a set of attenuation bands seen in the transmission spectrum of the optical fiber core. The study of the LPG attenuation bands has yielded many potential applications in the field of sensing through their sensitivities to strain $(\varepsilon)$, temperature $(T)$, the refractive index of the surrounding medium $\left(n_{s}\right)$, and bending [1]-[9]. The LPGs sensitivity to these parameters can manifest itself in two ways. First, the central wavelength of the attenuation band can shift in the spectral domain, which will be referred to as spectral sensitivity and, second, a change in the spectral transmission profile of the attenuation band may occur. The spectral shift of the attenuation band arises from the phase matching condition of the LPG [1].

A common problem with LPG-based sensors is discriminating between the various measurands. Various types of fiber

Manuscript received March 1, 2003; revised August 14, 2003.

T. Allsop, A. Gillooly, V. Mezentsev, D. J. Webb, and I. Bennion are with the Photonics Research Group, Aston University, Aston Triangle, Birmingham, U.K.

T. Earthgrowl-Gould is with the Clinical Biomedical Engineering Research Group, Aston University, Aston Triangle, Birmingham, U.K.

R. Neal is with the Department of Communications and Electrical Engineering, Facility of Technology, University of Plymouth, Plymouth, U.K.

Digital Object Identifier 10.1109/TIM.2003.821508 have been investigated to alleviate this problem: Examples include the use of air-clad [10] or progressive three-layered [11] fibers to desensitize the structure to changes in the refractive index of the surrounding medium and the use of three-layered W-fibers either to reduce sensitivity to temperature changes [12] or, alternatively, to maximize the temperature sensitivity [13].

This paper presents for the first time, to the authors' knowledge, a study of the spectral characteristics of LPGs written in D-shaped single mode fiber to form bend sensors. The evolution of the LPGs attenuation bands under increasing curvature differs from normal step-index fiber, where splitting is observed due to induced birefringence. In the $\mathrm{D}$-shaped fiber, we observed no such splitting but new bands were seen to appear under the influence of bending. It is found that the wavelength separation between adjacent bend induced attenuation bands is approximately linear as a function of curvature. Furthermore, red and blue wavelength shifts were observed as the sensor was rotated at constant curvature.

Finally, a normal LPG attenuation band and a bend induced attenuation band are used to discriminate between temperature and bending effects, giving a well-conditioned sensitivity matrix that is comparable to other discrimination approaches [14], [15].

\section{FABRICATION OF GRATING DEVICES}

The D-shaped single mode fiber was originally designed for coupler fabrication. The core's radius is $4 \mu \mathrm{m}$, and the distance between its center and the flat of the $\mathrm{D}$ is $9 \mu \mathrm{m}$ with a cladding radius of $62.5 \mu \mathrm{m}$. The center of the core is located at the center of the curved section of claddings radius. The core is a composition of $\mathrm{GeO}_{2} / \mathrm{SiO}_{2}$ and the cladding is assumed to be $\mathrm{SiO}_{2}$. The D-fiber is not specifically designed to be photosensitive and so its photosensitivity was increased by hydrogenation at a pressure of 120 Bar for a period of two weeks. The LPGs were fabricated using a frequency doubled argon ion laser at a wavelength of $244 \mathrm{~nm}$ with a point-by-point writing technique. The orientation of the $\mathrm{D}$ shape was determined and tags were attached to the fiber to ensure there was no twist in the fiber during fabrication. Several grating periods were used from 140 to $400 \mu \mathrm{m}$ with a grating length of $5 \mathrm{~cm}$. The characterization of the attenuation band was carried out by illuminating the LPG using a broadband light source and observing the transmission spectrum with an optical spectrum analyzer (OSA) with an accuracy of $0.05 \mathrm{~nm}$.

Scrutinizing the transmission spectrum during fabrication, it was noticed that the attenuation bands grew in strength with a red wavelength shift but this strengthening and red wavelength 


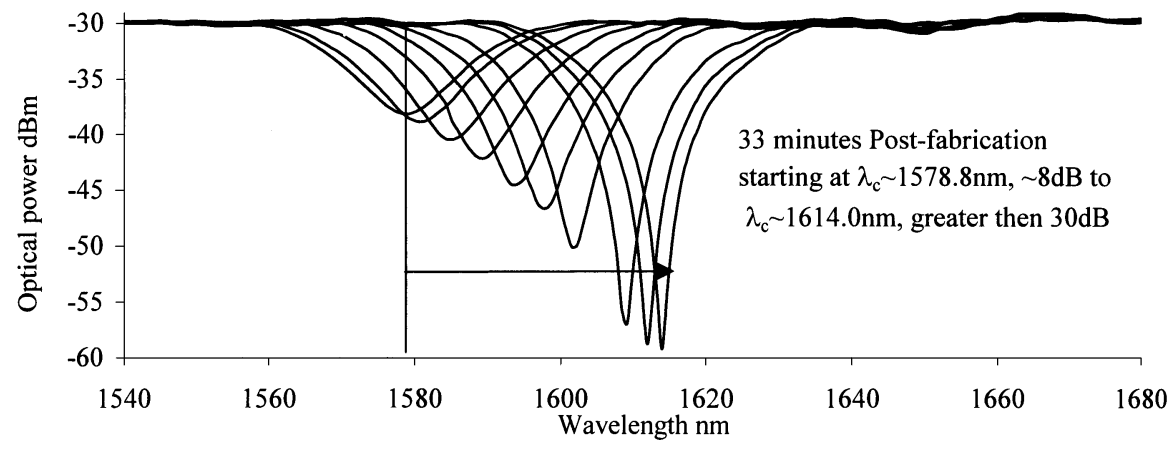

Fig. 1. Post-fabrication spectral evolution of an attenuation band from a LPG (period $=400 \times 10^{-6} \mathrm{~m}$, length $5 \mathrm{~cm}$ ) written in a D-shaped optical fiber.

shifting continued post-fabrication with red shifts well in excess of $150 \mathrm{~nm}$ followed by a roughly comparable blue wavelength shift. An example of part of the post-fabrication spectral evolution of the LPG attenuation is shown in Fig. 1. We hypothesize that this behavior is due to differing diffusion rates of hydrogen from the core and cladding, but this is to be the subject of future investigations.

\section{SPECTRAL Bending Characteristics}

The LPG (period $=380 \times 10^{-6} \mathrm{~m}$, length $5 \mathrm{~cm}$ ) was clamped mid-way between two towers; one of the clamps was mounted on a translation stage, which was moved inwards to induced a bend in the optical fiber. The tags used during fabrication and markers on the fiber were used to ensure there was no twist in the fiber during the experiment. Additionally, rotation of the LPG sensor was performed on this rig by subjecting the sensor to a known curvature then rotating the sensor around its clamped axis with the flat side always pointing upwards, defining as a $0^{\circ}$ reference the original orientation (fiber hanging downward, flat-side up); see Fig. 2.

The sensor's curvature $R$ is given by [8]

$$
R=\frac{2 \cdot d}{\left(d^{2}+L^{2}\right)}
$$

where $L$ is the half distance between the edges of the two towers, and $d$ is the bending displacement at the center of the LPG.

\section{A. Bending Characteristics}

The LPG sensor was subjected to a range of curvatures and the transmission spectra are shown in Fig. 3.

The bending experiment was repeated on a D-shaped fiber without an LPG and no significant change in the transmission spectrum of the fiber was observed over the range of curvatures used. Also over the bending range used there was no significant overall loss of transmission power through the D-fiber.

The polarization dependence of the attenuation bands was also observed. This was done by passing the broadband light source through a polarizer (Sifam single mode $1550 \mathrm{~nm}$ polarizer), a polarization controller and a 90:10 coupler, prior to injecting the light from the $90 \%$ port into the sensing fiber. The $10 \%$ port of the coupler was used to monitor the polarization state of the light with a polarimeter (Tektronix, PAT 9000B).

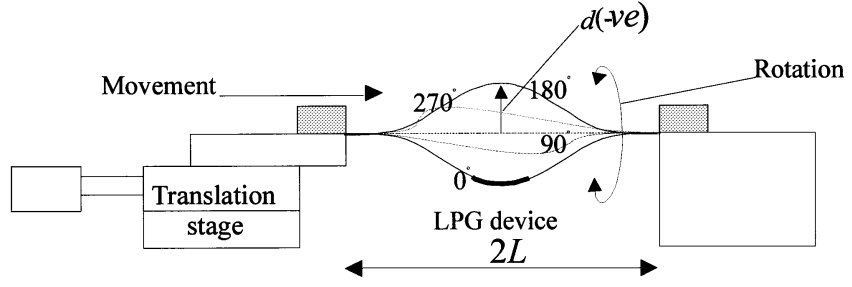

Fig. 2. Schematic of the bending/rotational test rig.

The LPG (period $=400 \times 10^{-6} \mathrm{~m}$, length $=5 \mathrm{~cm}$ ) was subjected to two curvatures $(R)$. First, the polarization behavior of a normal attenuation band of the LPG was investigated with $R=$ $1.8 \mathrm{~m}^{-1}$ which gave $\pm 0.5-\mathrm{nm}$ shift in the center wavelength and $\sim 5-\mathrm{dB}$ changes in strength due to polarization variations. Second, the behavior of a pair of the bend induced attenuation bands of the LPG was investigated with $R=4.5 \mathrm{~m}^{-1}$ which gave \pm 0.75 -nm and \pm 1.3 -nm shifts in the center wavelengths and $\sim 0.5-\mathrm{dB}$ changes in strength due to polarization variations. Other yet to be published evidence from compression loading of the LPG written in this D-shaped fiber shows no LPG attenuation band splitting due to birefringence [16]. This preliminary investigation into polarization suggests that the bend induced LPG attenuation bands are not caused by birefringence induced by bending, as described in [16]. Furthermore, the polarization state of the incident light has little effect on the observed spectral structure.

Fig. 4 shows the spectral sensitivity to bending of two attenuation bands-one normal and one bend induced. Both show an approximately linear response to curvature.

Over the curvature range of $0.5 \mathrm{~m}^{-1}$ to $4 \mathrm{~m}^{-1}$, we observed a total wavelength shift of $38 \mathrm{~nm}$ for the bend induced attenuation band, giving a bend sensitivity of $d \lambda / d R=12.55 \pm$ $2 \times 10^{-2} \mathrm{~nm} \cdot \mathrm{m}$ with the residuals having a standard deviation in curvature of $2 \times 10^{-2} \mathrm{~m}^{-1}$. The normal attenuation band had a sensitivity of $d \lambda / d R=-1.735 \pm 8 \times 10^{-3} \mathrm{~nm} \cdot \mathrm{m}$ with the residuals having a curvature standard deviation of $9 \times$ $10^{-} 3 \mathrm{~m}^{-1}$, the experimental results were also compared to theoretical predictions based upon the model described later in the text which showed reasonable agreement. Inspecting the bend induced attenuation bands, it was found that the spectral separation between adjacent bands (attenuation bands at 1280 and $1350 \mathrm{~nm}$ ) was an approximately linear function of curvature applied to the LPG sensor; see Fig. 5. 


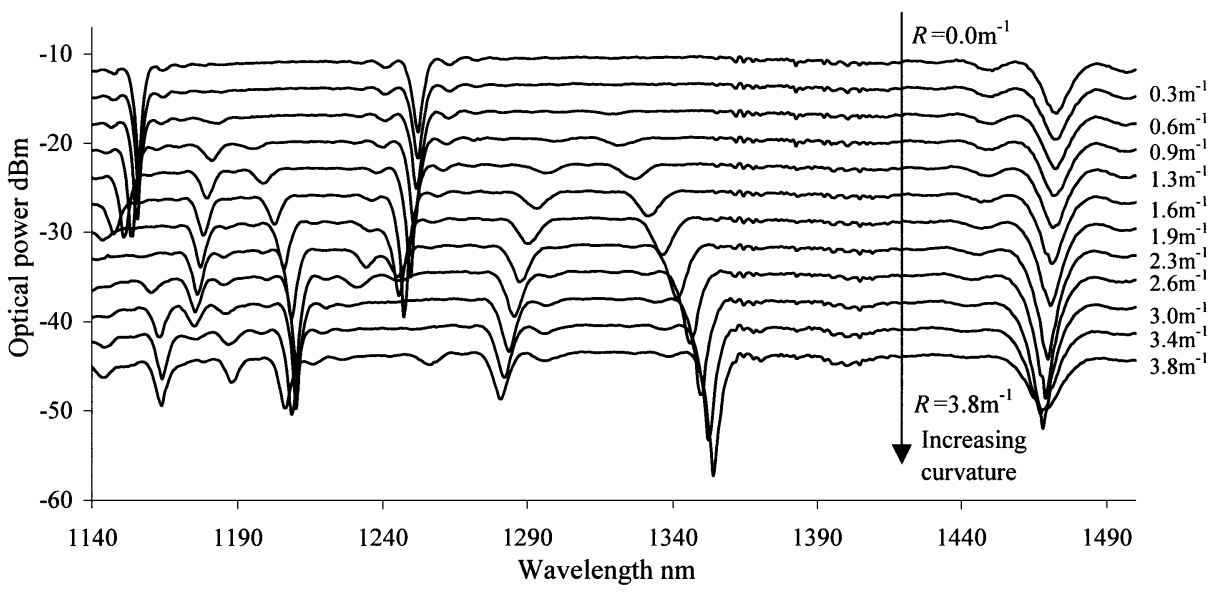

Fig. 3. Evolution of the transmission spectrum of the long period grating written in D-shaped fiber (period $=380 \times 10^{-6} \mathrm{~m}$, length $=5 \mathrm{~cm}$ ) subjected to bending, showing the appearance of bend induced stopbands.

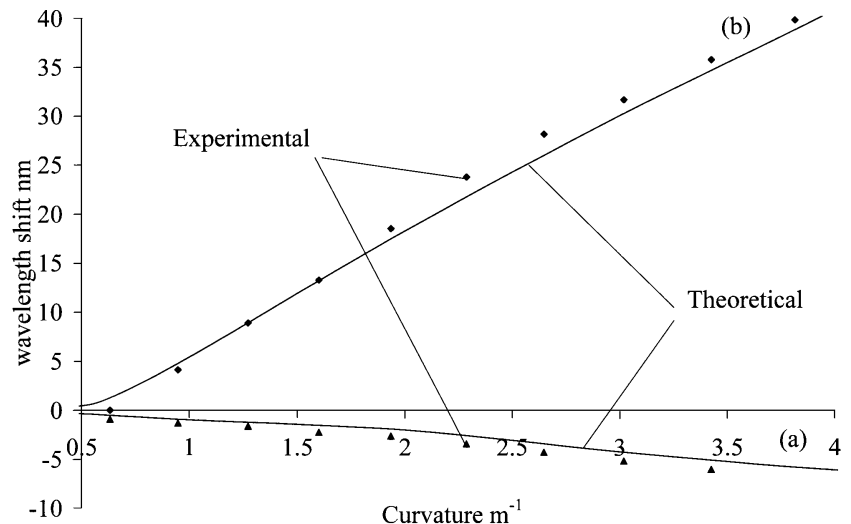

Fig. 4. Spectral sensitivity of a LPG (period $=380 \times 10^{-6} \mathrm{~m}$, length $\left.=5 \mathrm{~cm}\right)$ as a function of curvature for the attenuation band associated with (a) a normal stopband at $1470 \mathrm{~nm}$ and (b) a bend induced stopband at $1350 \mathrm{~nm}$ shown in Fig. 3.

\section{B. Rotation Characteristics}

The LPG was rotated through $360^{\circ}$ with a fixed curvature maintaining the flat of the D pointing upwards (see Fig. 1), which resulted in red and blue wavelength shifts of its attenuation bands, dependent upon the sense of rotation. This spectral behavior was noted for all normal and induced attenuation bands observed for LPGs with periods of $240 \times 10^{-6}, 340 \times 10^{-6}$, $380 \times 10^{-6}$, and $400 \times 10^{-6} \mathrm{~m}$; an example is shown in Fig. 6 .

The rotational experiment was repeated for a range of curvatures and it was found that the magnitude of the wavelength shift produced by rotation was linearly dependent upon the degree of curvature experienced by the LPG sensors; see Fig. 7.

Rotational wavelength shift similar to this has been observed before for an LPG in standard step-index fiber [7]. In the case of the D-shaped fiber, the curvature induces an index profile change and bending strain with the asymmetry causing tension or compression in the core region depending upon the orientation of the bend to flat of the D-fiber. Wavelength shifts have also been reported in an eccentric core fiber [9], which uses the asymmetry of the core's position away from the waveguide's central axis to induce tension and compression.

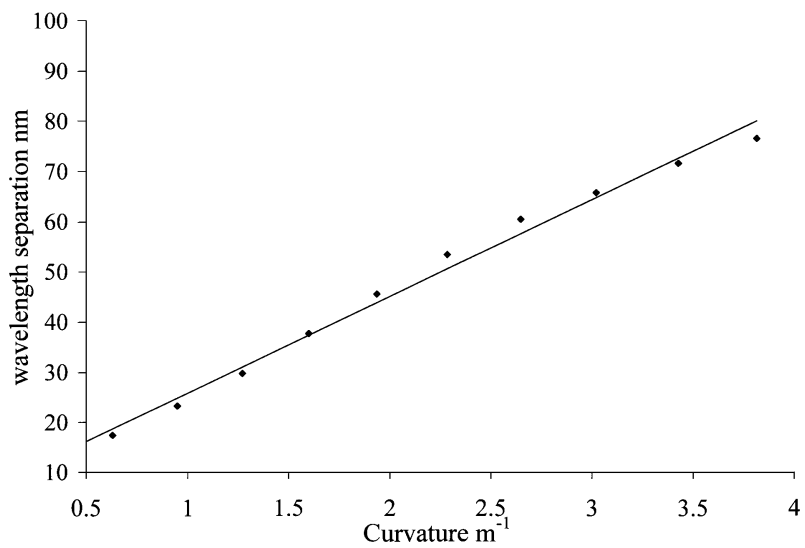

Fig. 5. Wavelength separation as a function of curvature for the bend-induced attenuation bands visible at 1280 and $1350 \mathrm{~nm}$, as shown in Fig. 3.

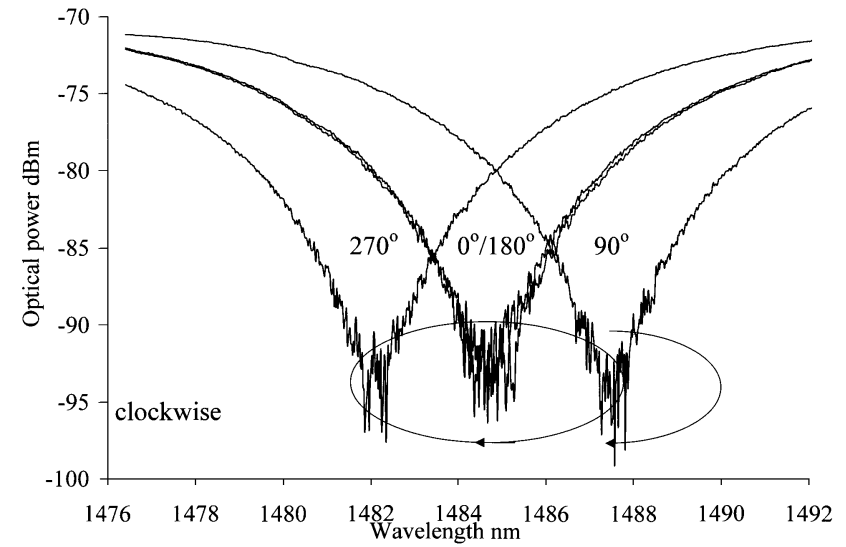

Fig. 6. Examples of the blue/red wavelength shifts experienced by one of the attenuation bands of the long period grating (period $=380 \times 10^{-6} \mathrm{~m}$, length $=5 \mathrm{~cm}$ ) written in the D-shaped fiber subjected to a rotation through $360^{\circ}$ at curvature of $2.7 \mathrm{~m}^{-1}$.

The waveguiding properties of the D-shaped optical fiber were investigated using a finite element method. To obtain the effects of bending on the attenuation bands, it is necessary to study the cladding modes associated with the attenuation bands. This is done in two parts. 
First, we need to obtain the mechanical effect on the radial variation of the longitudinal/lateral stress and strain for a given bend to obtain the refractive index profile of the fiber. This mechanical information was used in conjunction with the strainoptic coefficients of the core material $\left(d n_{\mathrm{co}} / d \varepsilon=2.8 \times 10^{-7}\right)$ and cladding material $\left(d n_{\mathrm{cl}} / d \varepsilon=2.4 \times 10^{-7}\right)$ to obtain index variation due to strain. The strain-optic coefficients of a glass material will vary with the composition and concentration of dopants but for simplicity we assume that these coefficients are constant for each layer of the optical fiber. The modeling gave the variation of the refractive index profile due to strain induced by the bending. As an example, it was found for a curvature of $5.5 \mathrm{~m}^{-1}$ the strain varied from +150 to $-150 \mu \varepsilon$ for a concave to a convex bend experienced by the LPG device.

Second, the bend/curvature of the D-fiber would change the properties of the waveguide itself and this was taken into account using conformal mapping. The conformal mapping technique replaces the curved optical fiber waveguide by an equivalent straight waveguide with the following index profile [17], [18] as a function of curvature $R$ and longitudinal strain $\varepsilon$

$$
n(R) \rightarrow\left(n(0)+\frac{d n}{d \varepsilon} \cdot \varepsilon\right) \cdot \exp \left(\frac{d}{R}\right)
$$

where $n(0)$ is the initial refractive index of the core/cladding, $R$ is the curvature experienced by the fiber and $d$ is the distance from the center of the fiber. After completing the above transforms, a finite element package (Femlab) was used to obtain the effective refractive indices of the core and the cladding modes at various curvatures (concave and convex bending) at a given wavelength. Also from this package the radial distributions of the E-field intensities across the D-fiber for individual cladding modes were obtained, and used to determine the coupling coefficients $(\kappa)$ for each cladding mode to the fundamental core mode. First, in the theoretical investigation, it was determined that the net increase in strain within the fiber induced by bending had a negligibly effect compared to the effects the bending had on the refractive index profile of the fiber itself.

The appearance of the additional attenuation bands under bending is due to the effect of bending on the coupling coefficients of the cladding modes. In the case of the straight D-fiber, it was found from the modeling that the attenuation bands present in the transmission spectrum are not consecutive in mode order, the $\kappa \mathrm{s}$ (overlap integrals) for cladding mode orders fourth, seventh, and tenth are significantly greater than for the first, fifth, etc. Therefore, only a small number of attenuation bands are present in the straight D-fiber case. When the D-fiber is bent the $\kappa \mathrm{s}$, for the fifth and sixth cladding modes, for example, become more significant, and their attenuation bands start to appear. The $\kappa$ s (overlap integrals) as a function of curvature for selected D-fiber cladding modes are shown in Fig. 8.

The coupling coefficients were calculated as normalized overlap integrals

$$
\kappa_{s}=\frac{\int \vec{E}_{0} \cdot \vec{E}_{s}^{*} d \vec{r}}{\sqrt{\int\left|\vec{E}_{0}\right|^{2} d \vec{r}} \sqrt{\int\left|\vec{E}_{s}\right|^{2} d \vec{r}}}
$$

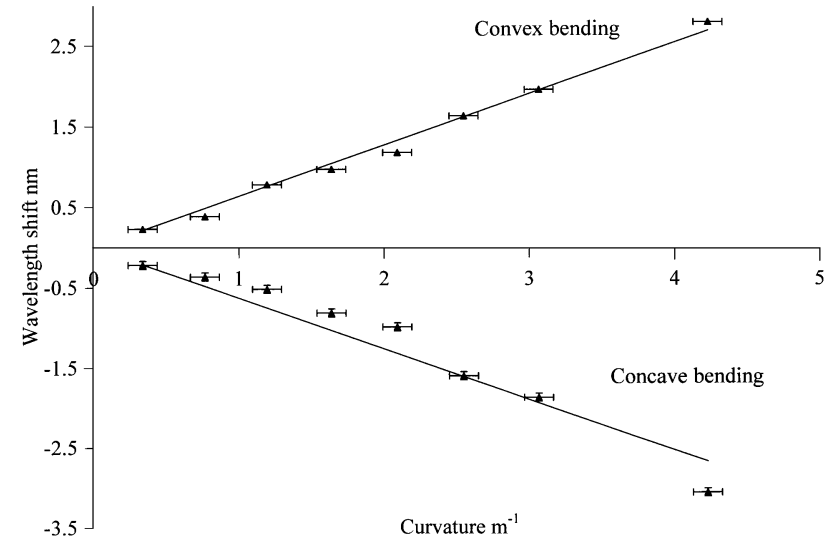

Fig. 7. Maximum observed displacement of an attenuation band centered at $1485 \mathrm{~nm}$ (LPG with a period $380 \times 10^{-6} \mathrm{~m}$ and length $5 \mathrm{~cm}$ ) during rotation through $360^{\circ}$ as a function of curvature.

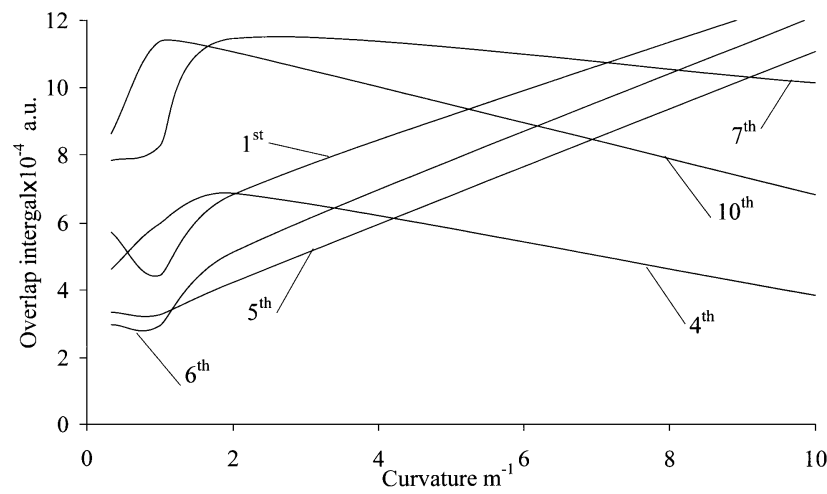

Fig. 8. Overlapping integrals between different cladding modes and the core mode at a wavelength of $1500 \mathrm{~nm}$ as a function curvature.

where $E_{0}$ and $E_{s}$ are transverse components of electric field of the core and $s$ th cladding mode correspondingly. Waveforms for all core and cladding modes were found using a full-vectorial finite element eigenvalue solver FEMLAB [19] for the waveguide geometry equivalent to D-shaped fiber with a core radius $4 \mu \mathrm{m}$ and distance between core center and flat surface $9 \mu \mathrm{m}$.

The orientational characteristics of these LPG attenuation bands were also investigated theoretically using the finite element package. The package was used to obtain the effective refractive indices of the core and cladding modes and their group indices at rotational angles of $0^{\circ}$ (a concave bend) and $180^{\circ}$ (a convex bend). This was achieved by using a second conformal mapping transform to describe the bend at a rotational angle of $180^{\circ}$. Inspecting the effective refractive index of the fourth cladding mode as a function of the curvature of the concave and convex bends, it was found that at the $0^{\circ}$ rotational position the effective refractive index increases with curvature while at $180^{\circ}$ the index decreases, with in both cases the core mode showing little variation, which explains the blue and red wavelength shifts observed. The theoretical wavelength shift was obtained using the same approach as [4] and [6] and gives

$$
\Delta \lambda=\left[\frac{\lambda}{\left(\delta n_{\mathrm{eff}}-\delta n_{g}\right)} \cdot \frac{d \delta n_{\mathrm{eff}}}{d R}+\frac{\left(\delta n_{\mathrm{eff}}\right)^{3}}{\delta n_{g}} \frac{d \Lambda}{d R}\right] \cdot \Delta R
$$

where $\delta n_{\text {eff }}=n_{\text {co eff }}-n_{\text {cleff }_{\nu}}$ is the differential effective index between the 4 th cladding mode and the core mode and 


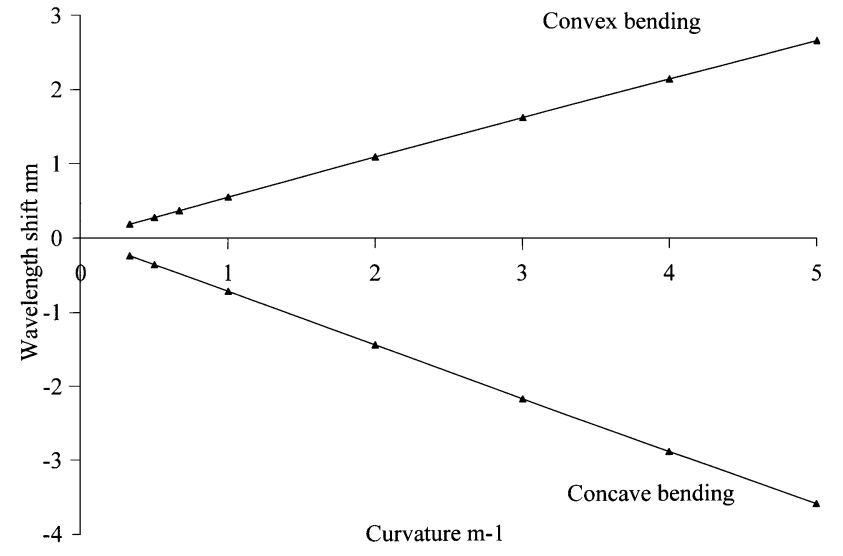

Fig. 9. Theoretical wavelength shifts of the attenuation band associated with the 4 th cladding mode being subjected to bending.

$\delta n_{g}=n_{\mathrm{co} g}-n_{\mathrm{clg}_{\nu}}$ is the differential group index. The effective refractive indices of the core and fourth cladding mode were calculated as a function of curvature using the finite element package. Using (3), reasonable agreement was obtained with experimental data; compare Figs. 7 and 9.

\section{Temperature Characteristics}

The sensitivity to temperature was investigated by placing the sensor under a thermally insulating cover on a hot-plate. The measurement of the temperature of the hot-plate was achieved by using a probe with an accuracy of $\pm 0.1^{\circ} \mathrm{C}$. The spectral locations of the central wavelengths of the attenuation bands were monitored. The temperature sensitivity experiment was repeated several times with various bending curvatures applied to the LPG device, typical results are shown in Fig. 10.

This LPG device (period $=380 \times 10^{-6} \mathrm{~m}$ and length $=5 \mathrm{~cm}$ ) can be used to discriminate between temperature and bending effects using the normal stopband at $1470 \mathrm{~nm}$ and the bend induced stopband at $1350 \mathrm{~nm}$, which give a well-conditioned sensitivity matrix

$$
\begin{aligned}
\left(\begin{array}{c}
\Delta \lambda_{1 \mathrm{st}} \\
\Delta \lambda_{2 \mathrm{nd}}
\end{array}\right)= & \left(\begin{array}{cc}
-1.735 \pm 8 \times 10^{-3} & 0.078 \pm 2 \times 10^{-3} \\
12.55 \pm 2 \times 10^{-2} & 0.022 \pm 6 \times 10^{-3}
\end{array}\right) \\
& \cdot\left(\begin{array}{c}
\Delta R \\
\Delta T
\end{array}\right)
\end{aligned}
$$

This sensitivity matrix yields a condition number of about 180 which is comparable to that from other approaches to discrimination [14], [15].

To obtain the cross sensitivity between bending and temperature for this LPG sensor, a similar procedure was adopted to the one described in [20]. Using a Taylor expansion for the temperature and bending induced changes in central wavelength of attenuation band, and making the assumption that there is no axial strain being applied to the sensor, we have the following expression, which contains the cross sensitivity

$$
\lambda=\delta n_{\mathrm{eff}_{\nu}} \Lambda+\frac{\partial \lambda}{\partial T} \cdot \Delta T+\frac{\partial \lambda}{\partial R} \cdot \Delta R+\frac{\partial^{2} \lambda}{\partial R \partial T} \cdot \Delta T \Delta R .
$$

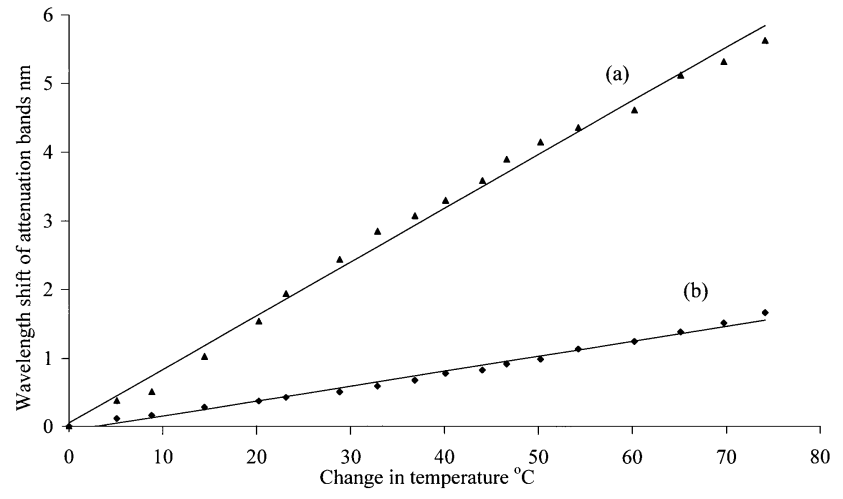

Fig. 10. Spectral sensitivity of the attenuation band associated with (a) normal stopband at $1470 \mathrm{~nm}$ and (b) the bend induced stopband at $1350 \mathrm{~nm}$ with the LPG at a curvature of $3.4 \mathrm{~m}^{-1}$ as a function of temperature (LPG with a period $380 \times 10^{-6} \mathrm{~m}$ and length $5 \mathrm{~cm}$ ).

Using the expression given in [4] for $(d \lambda / d T)$ and differentiating with respect to curvature $R$ yields the following expression for the spectral cross sensitivity of this LPG sensor:

$$
\begin{aligned}
\frac{\partial^{2} \lambda}{\partial T \partial R}= & \frac{-\Delta \xi \cdot \lambda}{\left(\delta n_{\mathrm{eff}}-\delta n_{g}\right)^{2}} \cdot\left[\frac{\partial \delta n_{\mathrm{eff}}}{\partial R}-\frac{\partial \delta n_{g}}{\partial R}\right] \\
& +\Lambda \alpha \cdot\left[2 \frac{\delta n_{\mathrm{eff}}}{\delta n_{g}} \cdot \frac{\partial \delta n_{\mathrm{eff}}}{\partial R}-\left(\frac{\delta n_{\mathrm{eff}}}{\delta n_{g}}\right)^{2} \cdot \frac{\partial \delta n_{g}}{\partial R}\right] \\
& +\alpha \cdot \frac{\left(\delta n_{\mathrm{eff}}\right)^{2}}{\delta n_{g}} \cdot \frac{\partial \Lambda}{\partial R} .
\end{aligned}
$$

Some of the terms in the above expression are already defined, the others are $\alpha$, the thermal expansion coefficient of the core and $\Delta \xi_{\nu}$, the differential thermo-optic coefficient between the core $\left(7.3 \times 10^{-6}{ }^{\circ} \mathrm{C}^{-1}\right)$ and the cladding material $\left(7.8 \times 10^{-6}{ }^{\circ} \mathrm{C}^{-1}\right)$ from [5]. We assume that the amount of birefringence induced is negligibly small for curvatures of $2 \mathrm{~m}^{-1}$ (no splitting of the attenuation bend was observed experimentally over the range of curvatures studied). Using the results obtained from the finite element package the spectral cross-sensitivity was calculated to be $1.3 \times 10^{-4} \mathrm{~nm} \mathrm{~m}{ }^{\circ} \mathrm{C}^{-1}$. This figure is small and over the experimental measurement range gives a maximum relative cross sensitivity error of about $0.4 \%{ }^{\circ} \mathrm{C}^{-1}$.

\section{CONCLUSION}

Long period gratings have been written in D-shaped optical fiber where their spectral sensitivities to bending and temperature have been investigated. The dependence of their transmission spectra with curvature is distinct from step-index fiber: as curvature is increased, more attenuation bands appear in the spectrum of the LPG. These transmission features are due to mode coupling coefficients that increase with curvature. Also, it was found that these attenuation bands were sensitive to the orientation of the bend with respect to the flat of the $\mathrm{D}$; thus, this LPG device could, in principle, be used as a directional bend sensor.

This LPG sensor can also be used to discriminate between temperature and bending effects and yields a reasonably wellconditioned sensitivity matrix, comparable to those obtained in other sensing schemes. 


\section{REFERENCES}

[1] A. Vengsarkar et al., "Long-period gratings as band-rejection filters," $J$. Lightwave Technol., vol. 14, pp. 58-64, Jan. 1996.

[2] V. Bhatia and A. M. Vengsarkar, "Optical fiber long-period grating sensors," Opt. Lett., vol. 21, no. 9, pp. 692-694, 1996.

[3] H. J. Patrick, A. D. Kersey, and F. Bucholtz, "Analysis of the response of long period fiber gratings to external index of refraction," J. Lightwave Technol., vol. 16, pp. 1606-1612, Sept. 1998.

[4] V. Bhatia, "Applications of long-period gratings to single and multi-parameter sensing," Opt. Express., vol. 4, no. 11, pp. 457-466, 1999.

[5] X. Shu et al., "Room-temperature operation of widely tunable loss filter," Electron. Lett., vol. 37, no. 4, pp. 216-218, 2001.

[6] T. Allsop et al., "Detection of organic aromatic compounds in paraffin by a long period fiber grating optical sensor with optimized sensitivity," Opt. Commun., vol. 191, pp. 181-190, 2001

[7] H. J. Patrick, C. C. Chang, and S. T. Vohra, "Long period fiber gratings for structural bend sensing," Electron. Lett., vol. 34, no. 18, pp. 1773-1775, 1998.

[8] W. Du, H. Tam, M. Liu, and X. Tao, "Long-period fiber grating bending sensors in laminated composite structures," in Proc. Sensory Phenomena and Measurement Instrumentation for Smart Structures and Materials, 1998, pp. 284-292.

[9] H. J. Patrick, "Self-aligning, bipolar bend transducer based on long period grating written in eccentric core fiber," Electron. Lett., vol. 36, no. 21, p. 1763-1764, 2000.

[10] R. P. Espindola et al., "External refractive index insensitive air-clad long period fiber grating," Electron. Lett., vol. 35, no. 4, pp. 327-328, 1999.

[11] T. Allsop et al., "Investigations of the spectral sensitivity of long period gratings fabricated in 3-layered optical fiber," J. Lightwave Technol. to be published.

[12] J. Jang et al., "Temperature insensitive long-period fiber gratings," Electron. Lett., vol. 35, no. 24, pp. 2134-2136, 1999.

[13] S. Yin et al., "A highly sensitive long period grating based tunable filter using a unique double-cladding layer structure," Opt. Commun., vol. 188, pp. 301-305, 2001.

[14] H. Patrick, G. Williams, A. Kersey, J. Pedrazzani, and A. Vengsarkar, "Hybrid fiber bragg grating/long period fiber grating sensor for strain/temperature discrimination," Photon. Technol. Lett., vol. 8, no. 9, pp. 1223-1225.

[15] M. G. Xu, J.-L. Archambault, L. Reekie, and J. P. Dakin, "Discrimination between strain and temperature effects using dual-wavelength fiber grating sensors," Electron. Lett., vol. 30, no. 13, pp. 1085-1087, 1994.
[16] L. Zhang et al., "Design and realization of long-period grating devices in conventional and high birefringence fibers and their novel applications as a fiber-optic load sensors," IEEE J. Select. Topics in Quantum Electron., vol. 5, pp. 1373-1378, 1999.

[17] M. Heiblum and J. H. Harris, "Analysis of curved optical waveguides by conformal transformation," IEEE J. Quantum Electron., vol. QE-11, no. 2 , pp. $75-83,1975$

[18] F. Muhammad et al., "Analysis of curved D-fiber for Methane gas sensing," Photon. Technol. Lett., vol. 7, no. 5, pp. 538-539, 1995.

[19] K. Eriksson, D. Estep, P. Hansbo, and C. Johnson, Computational Differential Equations. Cambridge, U.K.: Cambridge Univ. Press, 1996.

[20] J. Ma et al., "Optical-fiber sensor for simultaneous measurement of pressure and temperature: Analysis of cross sensitivity," Appl. Opt., vol. 35 , no. 25 , pp. 5206-5210, 1996.

T. Allsop, photograph and biography not available at the time of publication.

A. Gillooly, photograph and biography not available at the time of publication.

V. Mezentsev, photograph and biography not available at the time of publication.

T. Earthgrowl-Gould, photograph and biography not available at the time of publication.

R. Neal, photograph and biography not available at the time of publication.

D. J. Webb, photograph and biography not available at the time of publication.

I. Bennion, photograph and biography not available at the time of publication. 